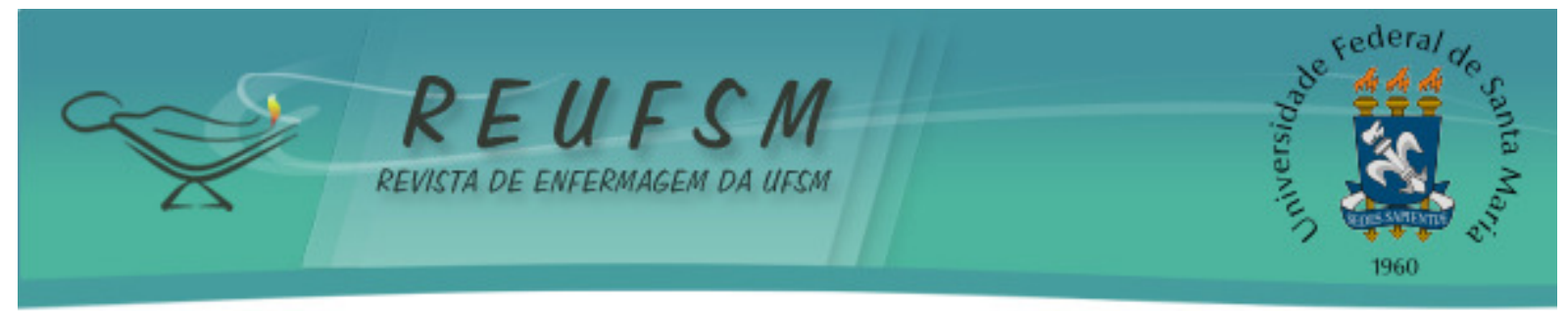

ARTIGO DE REVISÃO

\title{
ADESÃO AO TRATAMENTO NA HIPERTENSÃO E DIABETES MELLITUS: REVISÃO INTEGRATIVA
}

\section{TREATMENT ADHERENCE IN HYPERTENSION AND DIABETES MELLITUS: AN INTEGRATIVE REVIEW ADHESIÓN AL TRATAMENTO DE HIPERTENSIÓN Y DIABETES MELLITUS: UNA REVISIÓN INTEGRADORA}

Doi: $10.5902 / 2179769219029$

\author{
Aline Moraes de Abreu ${ }^{1}$ \\ Nikolas Paim Brandão \\ Sonara Lúcia Estima ${ }^{3}$ \\ Michelli Cristina Silva de Assis ${ }^{4}$
}

RESUMO: Objetivo: analisar as evidências disponíveis na literatura sobre as causas de adesão ou não ao tratamento de pacientes hipertensos e/ou diabéticos. Método: revisão integrativa, que selecionou artigos publicados entre 2005 e 2015, localizados por meio dos descritores "adesão à medicação", "cooperação do paciente", "diabetes mellitus tipo 2", "enfermagem" e "hipertensão". As bases consultadas foram Biblioteca Virtual em Saúde (Enfermagem), LILACS e PubMed. Resultados: selecionou-se 26 artigos, fatores associados à adesão foram: menor idade, ter estilo de vida saudável e itens relacionados à terapêutica. Dentre os fatores que levaram à pior adesão, destaca-se menor renda, pouco conhecimento sobre a doença e tratamento complexo. Conclusões: os fatores de adesão ao tratamento na hipertensão e diabetes foram relacionados ao paciente, ao tipo de terapêutica e ao sistema de saúde; os quais se constituem em importantes questões a serem abordadas pelos enfermeiros.

Descritores: Adesão à medicação; Cooperação do paciente; Diabetes mellitus tipo 2; Enfermagem; Hipertensão.

ABSTRACT: Aim: to analyze the scientific literature on the main causes of adherence or not to the treatment of hypertension and type 2 diabetes mellitus. Method: an integrative literature review, which selected full articles published between 2005 and 2015, detected by the descriptors "medication adherence", "patient compliance"," type 2 diabetes mellitus", "hypertension" and "nursing". The searches were conducted in the Virtual Health Library (Nursing), LILACS and PubMed databases. Results: Twenty-six articles were selected, the factors associated with adherence were: young age, to have a healthy lifestyle and items related to therapy. On the other hand, among the factors that lead to poor adherence, there are lower income, little knowledge about the disease and complex treatment. Conclusions: the adherence factors to the treatment of hypertension and diabetes are related to the patient, to the type of treatment and health system, which constitute important issues to be approached by nurses.

Descriptors: Medication adherence; Patient compliance; Diabetes mellitus type 2; Nursing; Hypertension.

\footnotetext{
${ }^{1}$ Enfermeira. Santa Casa de Misericórdia de Porto Alegre. Porto Alegre, Rio Grande do Sul, Brasil. Email: alineurug@yahoo.com.br.

2 Acadêmico de Enfermagem. Centro Universitário Lasalle. Canoas, Rio Grande do Sul, Brasil. Email: nikolas_brandao@hotmail.com.

${ }^{3}$ Enfermeira. Doutora em Educação. Centro Universitário Lasalle. Canoas, Rio Grande do Sul, Brasil. Email: sonara.estima@unilasalle.edu.br.

${ }^{4}$ Enfermeira. Doutora em Ciências Médicas. Centro Universitário Lasalle. Canoas, Rio Grande do Sul, Brasil. Email: michellicassis@gmail.com
} 


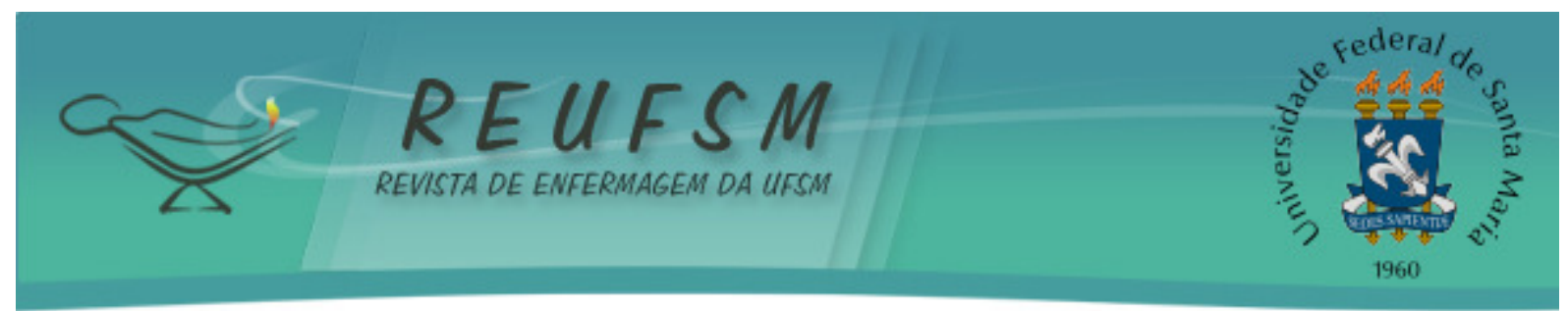

RESUMEN: Objetivo: Analizar la producción científica de las causas principales de la adhesión o no al tratamiento de los pacientes con hipertensión y diabetes mellitus tipo 2. Método: revisión integradora, que seleccionó artículos completos publicados entre 2005 y 2015, con los descriptores: "Cumplimiento de la medicación"; "Cooperación del paciente"; "Diabetes mellitus tipo 2"; "Hipertensión", "Enfermería", en las bases de datos Biblioteca Virtual en Salud (Enfermería), LILACS y PubMed. Resultados: Se seleccionaron 26 artículos sobre los factores asociados a la adhesión fueron: menores de edad, estilo de vida saludable y elementos relacionados a la terapia. Entre los factores que llevaron a la baja adhesión hay menos ingresos, poco conocimiento sobre la enfermedad y el tratamiento complejo. Conclusiones: los factores de adhesión al tratamiento de la hipertensión y de la diabetes están relacionados al paciente, al tipo de tratamiento y al sistema de salud, que constituyen importantes cuestiones que deben ser abordadas por los enfermeros.

Descriptores: Cumplimiento de la medicación; Cooperación del paciente; Diabetes mellitus tipo 2; Enfermería; Hipertensión.

\section{INTRODUÇÃO}

Entre as doenças crônicas, a hipertensão arterial sistêmica (HAS) se destaca como a mais comum e perigosa, por ser assintomática até estágios muito evoluídos, podendo gerar complicações cardiovasculares. ${ }^{1}$ Observações clínicas em progresso demonstram que existe grande risco de o diabetes mellitus tipo 2 (DM2), que também é uma doença crônica, ser associado à hipertensão. Acerca deste assunto, a possibilidade de associação das duas doenças é da ordem de 50\%, o que requer, na maioria dos casos, o manejo de ambas em um mesmo paciente. ${ }^{2}$

Essas doenças, consideradas como fatores de risco pela elevação das taxas de morbimortalidade, se tornam ainda mais problemáticas e não controláveis quando a não adesão ao tratamento é baixa. Adesão é definida como a situação na qual o comportamento do paciente corresponde ao conselho médico ou de saúde, pode ser avaliada pelo comparecimento às consultas, a tomada de medicações ou pelas mudanças de estilo de vida. ${ }^{3}$ No caso de pacientes hipertensos e diabéticos, o tratamento engloba tanto a tomada de medicamentos orais ou injetáveis (manejo farmacológico) e mudanças de estilo de vida, como a prática de atividade física e alimentação saudável (manejo não farmacológico). ${ }^{4}$ Portanto, a má adesão a ambos tipos de tratamento pode colaborar para o aumento dos índices de morbidades, internações e dos custos no sistema de saúde. ${ }^{1,5}$ Estudo avaliou 385 hipertensos adultos da atenção primária e verificou que a adesão ao tratamento farmacológico foi de $59 \%{ }^{4}$ Por outro lado, existem dados de que para os diabéticos as taxas de adesão oscilam de 31 a $98 \%$ conforme a complexidade do tratamento. ${ }^{6}$

Destaca-se, por meio de meta-análise, estudos que avaliaram o efeito do exercício (tratamento não farmacológico) no controle glicêmico e na composição corporal, e os efeitos do exercício no condicionamento cardiorrespiratório de adultos com DM2. ${ }^{7}$ Os autores encontraram uma redução no controle glicêmico pósintervenção de $0,66 \%$ e redução de $11 \%$ no peso corporal. Também, os pesquisadores verificaram que o exercício aeróbico aumentou cerca de 9,5\% o condicionamento cardiorrespiratório. ${ }^{7,8}$

0 enfermeiro, atuando na equipe multidisciplinar, pode substancialmente contribuir com medidas e intervenções que possam melhorar os índices de adesão ao tratamento, tendo em vista que os dados disponíveis na literatura revelam índices de adesão inferiores ao estipulado pela OMS, de no mínimo $80 \%{ }^{3}$ Além disso, não há estudos bem delineados 


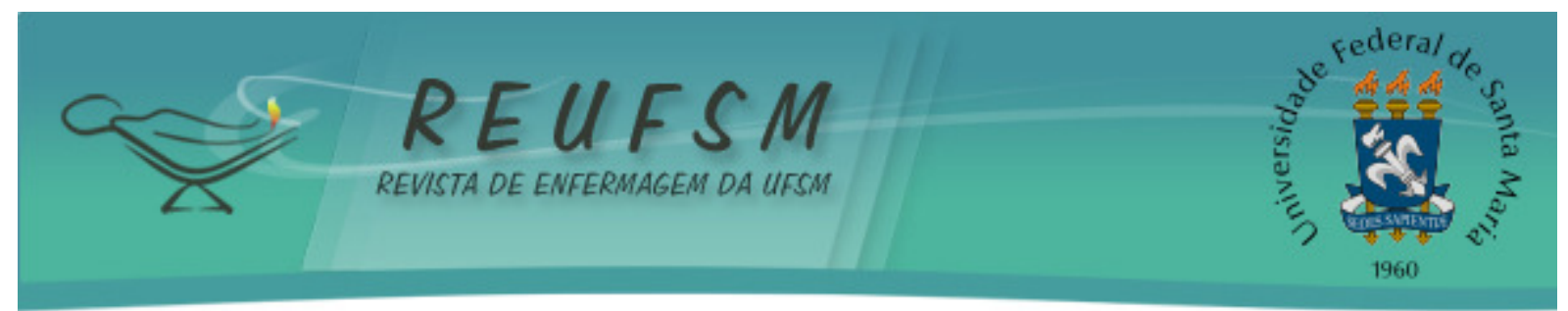

disponíveis que possam remeter à abordagem de enfermagem eficaz em melhorar este desfecho.

Frente a isto, identificou-se a necessidade de aprofundar o conhecimento sobre este assunto, para subsidiar o cuidado de enfermagem, utilizando-se da literatura disponível e obtendo ferramentas para implementar intervenções de enfermagem que possam qualificar e reduzir as complicações da hipertensão e DM2. Logo, uma revisão de literatura foi desenvolvida com o objetivo de analisar as evidências disponíveis na produção científica sobre as principais causas de adesão ou não ao tratamento de pacientes com hipertensão e DM2. A questão de pesquisa foi: quais são as principais causas de adesão e não adesão ao tratamento de pacientes com hipertensão e diabetes mellitus tipo 2?

\section{MÉTODO}

Revisão integrativa desenvolvida nas seguintes etapas: estabelecimento da questão de pesquisa, revisão de literatura, categorização dos estudos, avaliação dos estudos, interpretação dos dados e síntese do conhecimento. ${ }^{9}$ As bases pesquisadas foram a Biblioteca Virtual em Saúde (BVS), Enfermagem, Literatura LatinoAmericana e do Caribe em Ciências da Saúde (LILACS) e o PubMed. Para a coleta de dados dos artigos, finalizada em agosto de 2016, utilizou-se um instrumento em que constava referência completa do periódico, descritores, objetivos, método, participantes, resultados e conclusões. Os critérios de inclusão considerados foram: estudos publicados em português, inglês ou espanhol entre 2005 e 2015, estudos sobre a adesão aos tratamentos farmacológicos e não-farmacológicos de pacientes hipertensos e/ou diabéticos (tipo 2), e estar totalmente disponível online. 0 recorte temporal foi definido tendo como base a primeira publicação do Ministério da Saúde em $2006^{10}$ e a de $2013^{11}$, que versam sobre o papel do enfermeiro em abordar a adesão ao tratamento medicamentoso e não medicamentoso na hipertensão e DM2, respectivamente.

Os descritores utilizados foram: "adesão à medicação", "cooperação do paciente", “diabetes mellitus tipo 2", “enfermagem" e "hipertensão", em diferentes combinações com uso do operador booleano and. Foram excluídos estudos qualitativos, baseados em recomendações de guidelines. A análise crítica dos estudos incluídos foi realizada por dois autores independentes, seguida por um consenso entre os mesmos. A pesquisa inicial resultou em 72 artigos, após a leitura dos títulos e resumos disponíveis 32 foram excluídos, pois não respondiam aos critérios de inclusão do estudo. Quarenta artigos foram selecionados e lidos na íntegra, sendo sete na base de dados BVS, 46 na PubMed e 19 na LILACS (Figura 1), porém 14 apareceram em duplicata, restando para a análise final 26 artigos que foram interpretados pelos autores e sintetizados no instrumento de coleta dos dados segundo o nível de evidência proposto por Galvão (2003). ${ }^{12}$ Os aspectos éticos foram respeitados, uma vez que todos os autores consultados foram mencionados no texto. 

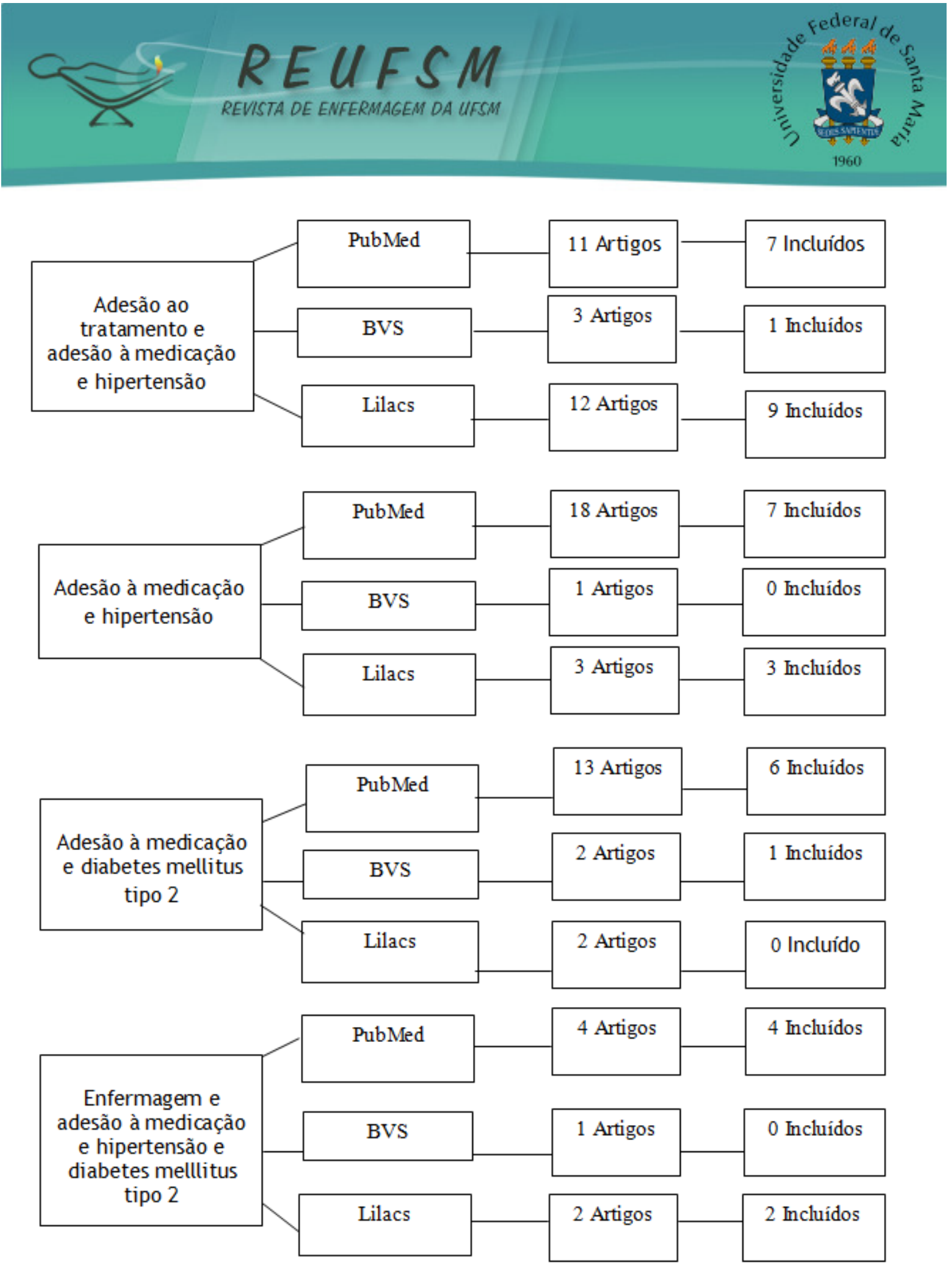

Figura 1 - Seleção dos artigos por agrupamento de descritores nas bases de dados. Canoas, RS, 2015.

\section{RESULTADOS E DISCUSSÃO}

A amostra final consistiu em 26 artigos, a maioria $(61,5 \%)$ estudos transversais e $23,1 \%$ randomizados. De 19 (73,0\%) artigos publicados em periódicos internacionais, 18 foram nos últimos cinco anos (Tabela 1). 


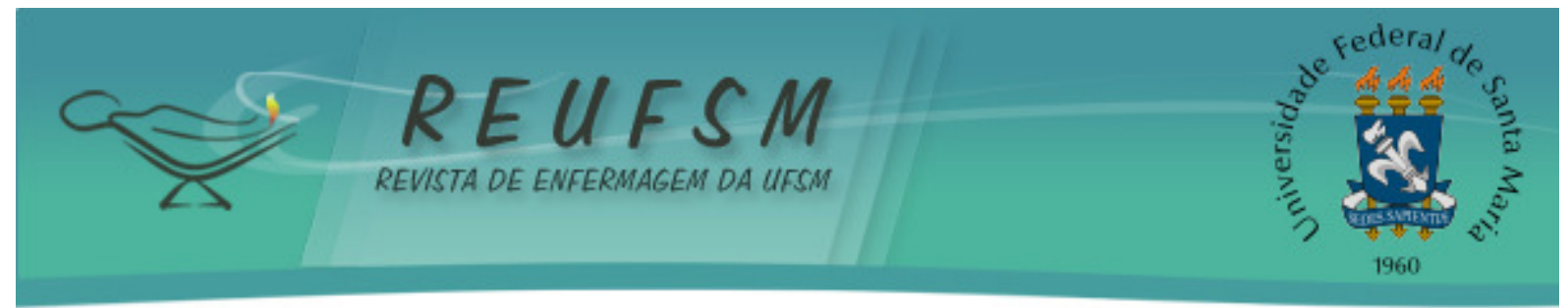

De modo geral, os objetivos propostos pelos estudos foram investigar a prevalência e fatores associados a não adesão ao tratamento medicamentoso em indivíduos, adultos ou idosos, com hipertensão e/ou diabetes em localidades do Brasil e em outros continentes; validar e verificar a performance de instrumentos que avaliam adesão; identificar se fatores demográficos, socioeconômicos ou culturais estão associados com adesão ao tratamento farmacológico e não-farmacológico; e verificar a importância e efetividade de estratégia para melhorar a adesão ao tratamento como um todo.

Tabela 1. Análise dos artigos quanto ao método, ano de publicação, periódico e nível de evidência.

\begin{tabular}{cccccc}
\hline Metodologia & $\begin{array}{c}\text { Ano de } \\
\text { publicação }\end{array}$ & $\begin{array}{c}\text { Periódico } \\
\text { brasileiro }\end{array}$ & $\begin{array}{c}\text { Periódico } \\
\text { internacional }\end{array}$ & $\begin{array}{c}\text { Nível de } \\
\text { evidência }\end{array}$ & $\begin{array}{c}\text { Total de } \\
\text { artigos } \\
\mathrm{N}(\%)\end{array}$ \\
\hline Retrospectivo & $2005-2010$ & 0 & 0 & 4 & $3(11,5)$ \\
& $2011-2015$ & 0 & 3 & & \\
Transversal & $2005-2010$ & 1 & 1 & 4 & $16(61,5)$ \\
& $2011-2015$ & 5 & 9 & & \\
Prospectivo & $2005-2010$ & 0 & 0 & 4 & $1(3,9)$ \\
& $2011-2015$ & 0 & 1 & & \\
Randomizado & $2005-2010$ & 0 & 0 & 2 & $6(23,1)$ \\
& $2011-2015$ & 1 & 5 & & \\
\hline Total N (\%) & $7(27,0)$ & $19(73,0)$ & & $26(100)$ \\
\hline
\end{tabular}

Os estudos consultados foram organizados por autores, fatores que melhoram ou pioram a adesão, tipo de paciente e base de dados consultada. (Quadro 1).

\begin{tabular}{|c|c|c|c|c|}
\hline Autores & $\begin{array}{l}\text { Fatores que melhoram } \\
\text { adesão }\end{array}$ & $\begin{array}{l}\text { Fatores que } \\
\text { adesão }\end{array}$ pioram & $\begin{array}{ll}\text { Tipo } & \text { de } \\
\text { paciente } & \end{array}$ & $\begin{array}{ll}\text { Base } \\
\text { dados }\end{array}$ \\
\hline $\begin{array}{l}\text { Ferreira RA, } \\
\text { Barreto SM, } \\
\text { Giatti L. }{ }^{13}\end{array}$ & $\begin{array}{l}\text { Aumento da idade e da } \\
\text { renda per capita. }\end{array}$ & $\begin{array}{l}\text { Sexo masculino, residir } \\
\text { nas regiões Norte, } \\
\text { Nordeste, e hábito de } \\
\text { fumar. }\end{array}$ & Hipertensos & PubMed \\
\hline $\begin{array}{l}\text { Blair IV, Steiner } \\
\text { JF, Hanratty R, et al. }{ }^{38}\end{array}$ & Não apresentado. & $\begin{array}{l}\text { Hipótese de que etnia } \\
\text { (preto, branco e latino) } \\
\text { piora adesão não foi } \\
\text { confirmada. }\end{array}$ & Hipertensos & PubMed \\
\hline $\begin{array}{l}\text { Vacek JL, } \\
\text { Hunt SL, } \\
\text { Shireman T. }{ }^{20}\end{array}$ & Ser jovem. & Não apresentado. & Hipertensos & PubMed \\
\hline Karakurt P, Kasikçi M. ${ }^{30}$ & Não apresentado. & $\begin{array}{l}\text { Esquecimento, } \\
\text { ignorância da } \\
\text { medicação, pobreza, } \\
\text { aposentadoria. }\end{array}$ & Hipertensos & PubMed \\
\hline $\begin{array}{l}\text { Alhalaiqa F, } \\
\text { Kho D, } \\
\text { AH N,et al. }{ }^{25}\end{array}$ & $\begin{array}{l}\text { Grupos individuais com } \\
\text { sessões sobre adesão. }\end{array}$ & Não apresentado. & Hipertensos & PubMed \\
\hline $\begin{array}{l}\text { Ribeiro AG, } \\
\text { Ribeiro SMR, } \\
\text { Dias CMGC,et al. }{ }^{24}\end{array}$ & Orientação nutricional. & Não apresentado. & Hipertensos & PubMed \\
\hline
\end{tabular}




\begin{tabular}{|c|c|c|c|c|}
\hline $\begin{array}{l}\text { Girotto E, } \\
\text { Andrade SM, Cabrera } \\
\text { MAS, et al. }{ }^{4}\end{array}$ & $\begin{array}{l}\text { Não consumir bebidas } \\
\text { alcoólicas, idade entre } \\
50 \text { a } 79 \text { anos, consultas } \\
\text { regulares ao médico e } \\
\text { IAM prévio. }\end{array}$ & Não apresentado. & Hipertensos & PubMed \\
\hline $\begin{array}{l}\text { Anguloa MLG, Urangaa } \\
\text { PL, Martín y M. I, et al. }\end{array}$ & $\begin{array}{l}\text { Maior nível de } \\
\text { conhecimento da } \\
\text { doença e menor número } \\
\text { de medicações. }\end{array}$ & $\begin{array}{l}\text { Menor capacidade } \\
\text { cognitiva, } \\
\text { perda funcional. }\end{array}$ & $\begin{array}{l}\text { Hipertensos e } \\
\text { diabéticos. }\end{array}$ & PubMed \\
\hline $\begin{array}{l}\text { Klamerus M, KerrEA, } \\
\text { Bosworth HB, Schmittdiel } \\
\text { J, et al. }{ }^{34}\end{array}$ & Não apresentado. & $\begin{array}{llr}\text { Depressão } & \text { e } & \text { pressão } \\
\text { arterial } & & \text { sistólica } \\
\text { elevada. } & & \\
\end{array}$ & $\begin{array}{ll}\text { Diabéticos } & \mathrm{e} \\
\text { hipertensos } & \end{array}$ & PubMed \\
\hline $\begin{array}{l}\text { Irvin MR, Shimbo D, Mann } \\
\text { DM, } \\
\text { Reynolds, K, et al. }{ }^{32}\end{array}$ & Não apresentado. & $\begin{array}{lr}\text { Resistência } & \text { ao } \\
\text { tratamento, mulheres, } \\
\text { inatividade, sintomas } \\
\text { depressivos. }\end{array}$ & Hipertensos & PubMed \\
\hline $\begin{array}{l}\text { Fürthauer J, Flamm M, } \\
\text { Sönnichsen A. }{ }^{28}\end{array}$ & Educação em saúde. & Não apresentado. & $\begin{array}{l}\text { Diabéticos } \mathrm{e} \\
\text { hipertensos. }\end{array}$ & PubMed \\
\hline $\begin{array}{l}\text { Nataraja N, } \\
\text { Putnam W, Aarsen KV, et } \\
\text { al. }{ }^{17}\end{array}$ & $\begin{array}{lllr}\text { ldade } & \text { maior } & \text { que } & 55 \\
\text { anos, estilo de vida } \\
\text { saudável. }\end{array}$ & Não apresentado. & $\begin{array}{ll}\text { Diabéticos } & \mathrm{e} \\
\text { hipertensos } & \end{array}$ & PubMed \\
\hline $\begin{array}{l}\text { Crowley MJ, Grubber JM, } \\
\text { Olsen MK, et al. }{ }^{22}\end{array}$ & Dieta e exercício. & Não apresentado. & Hipertensos & PubMed \\
\hline $\begin{array}{l}\text { Barreto MS, Reiners AAO, } \\
\text { Marcon SS. }{ }^{35}\end{array}$ & Não apresentado. & $\begin{array}{l}\text { Farmacoterapia } \\
\text { complexa, pouco } \\
\text { conhecimento sobre a } \\
\text { doença e insatisfação } \\
\text { com o serviço de saúde. }\end{array}$ & Hipertensos & LILACS \\
\hline $\begin{array}{l}\text { Vitor AF, Monteiro FPM, } \\
\text { Morais HCC, et al. }{ }^{21}\end{array}$ & $\begin{array}{l}\text { Abstinência ao etilismo } \\
\text { e tabagismo. }\end{array}$ & Não apresentado. & Hipertensos & LILACS \\
\hline $\begin{array}{l}\text { Santa-Helena ET, Nemes } \\
\text { MIB, Neto JE. }{ }^{31}\end{array}$ & Não apresentado. & $\begin{array}{l}\text { Classes econômicas } \\
\text { C/D/E, início do } \\
\text { tratamento há menos } \\
\text { de } 03 \text { anos e presença } \\
\text { de transtorno mental. }\end{array}$ & Hipertensos & LILACS \\
\hline $\begin{array}{l}\text { Walker RJ, Smalls BL, } \\
\text { Hernadez-Tejada } \\
\text { Campbell JA, et al. }{ }^{37}\end{array}$ & Não apresentado. & $\begin{array}{l}\text { Má adesão à dieta e à } \\
\text { prática de atividade } \\
\text { física. }\end{array}$ & Diabéticos & PubMed \\
\hline $\begin{array}{l}\text { Faria HTG, Santos MA, } \\
\text { Arrelias CCA, et al. }{ }^{27}\end{array}$ & $\begin{array}{l}\text { Política de distribuição } \\
\text { gratuita de } \\
\text { medicamentos, } \\
\text { esquema medicamento } \\
\text { fácil. }\end{array}$ & Não apresentado. & Diabéticos & PubMed \\
\hline $\begin{array}{l}\text { Bogner HR, Morales KH, } \\
\text { Vries HF, et al. }{ }^{33}\end{array}$ & Não apresentado. & $\begin{array}{l}\text { Depressão, função, } \\
\text { cognitiva diminuída, } \\
\text { custo de medicamentos, } \\
\text { experiências com } \\
\text { efeitos colaterais dos } \\
\text { medicamentos. }\end{array}$ & Diabéticos & PubMed \\
\hline $\begin{array}{l}\text { Schroeder EB, Hanratty } \\
\text { R, Beaty BL, et al. }{ }^{14}\end{array}$ & $\begin{array}{l}\text { Sexo masculino e não } \\
\text { ser fumante. }\end{array}$ & Não apresentado. & $\begin{array}{ll}\text { Diabéticos } & \mathrm{e} \\
\text { hipertensos } & \end{array}$ & PubMed \\
\hline $\begin{array}{l}\text { Solomon A, Schoenthaler } \\
\text { A, Seixas A, et al. }{ }^{29}\end{array}$ & $\begin{array}{l}\text { Programa consistente } \\
\text { de incentivo a tomar } \\
\text { medicação. }\end{array}$ & Não apresentado. & Hipertensos & PubMed \\
\hline
\end{tabular}




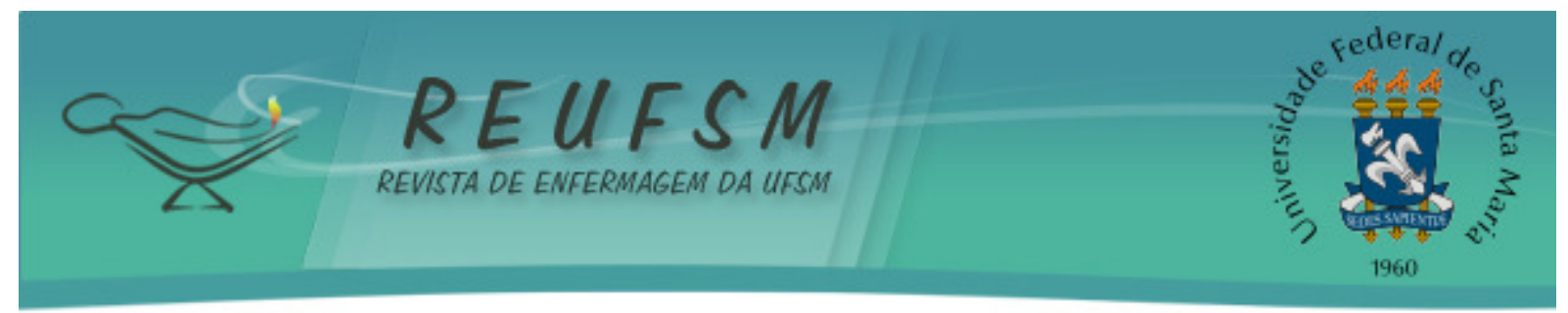

\begin{tabular}{|c|c|c|c|c|}
\hline Al-Ramahi R. ${ }^{18}$ & Não apresentado. & $\begin{array}{l}\text { Idade }<45 \text { anos, estado } \\
\text { de saúde regular } \\
\text { comparado ao } \\
\text { excelente, } \\
\text { esquecimento e efeitos } \\
\text { adversos. }\end{array}$ & Hipertensos & PubMed \\
\hline $\begin{array}{l}\text { Hedna K, Hakkarainen } \\
\text { KM, Gyllensten H, et al. }{ }^{26}\end{array}$ & Não apresentado. & $\begin{array}{l}\text { Novos usuários, uso de } 2 \\
\text { ou } \geq 3 \text { medicações anti- } \\
\text { hipertensivas. }\end{array}$ & Hipertensos & PubMed \\
\hline $\begin{array}{l}\text { Varleta P, Akel C, } \\
\text { Acevedo } \mathrm{M} \text {, et al. }{ }^{19}\end{array}$ & $\begin{array}{l}\text { Aumento da idade } \mathrm{e} \\
\text { estar desempregado. }\end{array}$ & $\begin{array}{l}\text { Esquecimento e efeitos } \\
\text { adversos. }\end{array}$ & $\begin{array}{ll}\text { Diabéticos } & \mathrm{e} \\
\text { hipertensos } & \end{array}$ & BVS \\
\hline $\begin{array}{l}\text { Lulebo AM, Mutombo PB, } \\
\text { Mapatano MA, et al. }{ }^{36}\end{array}$ & Não apresentado. & $\begin{array}{l}\text { Baixo conhecimento das } \\
\text { complicações da } \\
\text { hipertensão } \\
\text { medicações } \\
\text { indisponíveis no sistema } \\
\text { de saúde. }\end{array}$ & Hipertensos & PubMed \\
\hline $\begin{array}{l}\text { Ribeiro ÍJS; Boery RNS O, } \\
\text { Casotti CA, et al. }{ }^{15}\end{array}$ & $\begin{array}{l}\text { Situação marital de } \\
\text { união estável, controle } \\
\text { da hipertensão com } \\
\text { medicação e mudanças } \\
\text { no estilo de vida e } \\
\text { tempo de diagnóstico } \\
\text { inferior a } 10 \text { anos. }\end{array}$ & Não apresentado. & Hipertensos & BVS \\
\hline
\end{tabular}

Quadro 1. Síntese dos estudos incluídos na revisão integrativa, segundo fatores que melhoram ou pioram a adesão ao tratamento e sujeitos. PubMed, BVS e LILACS, 2005 a 2015. IAM- Infarto agudo do miocárdio; HAS - Hipertensão arterial sistêmica.

$\mathrm{Na}$ análise dos estudos, constatou-se que os fatores associados à adesão ao tratamento da HAS e DM2 podem ser divididos em fatores socioeconômicos: quanto maior a renda há melhora nos níveis de adesão em 57\%; ${ }^{13}$ fatores demográficos: ser do sexo masculino, ${ }^{14}$ ter situação marital de união estável, ${ }^{15}$ quanto maior a idade, entre 50 a 79 anos, ${ }^{4-16-19}$ porém em outro estudo ${ }^{20}$ ser jovem também melhora a adesão ao tratamento; fatores relacionados ao paciente: ter estilo de vida saudável ${ }^{15-17,21}$, o que inclui dieta pobre em sal e gordura, ${ }^{17-22}$ e prática de atividade física; ${ }^{22}$ ter tido IAM prévio ${ }^{16}$ e maior nível de conhecimento da doença; ${ }^{23}$ fatores relacionados à terapêutica: grupos de intervenção nutricional ${ }^{24}$ ou sessões individuais sobre adesão, ${ }^{25}$ menor número ${ }^{23}$ ou até sete medicações prescritas, ${ }^{17,26}$ esquema terapêutico fácil ${ }^{27}$ e tempo de diagnóstico inferior a dez anos; ${ }^{15}$ fatores relacionados ao sistema de saúde: consultas regulares ao médico, ${ }^{16}$ política de distribuição gratuita de medicamentos ${ }^{27}$ e estratégias de educação em saúde. ${ }^{28,}{ }^{29}$

Por outro lado, os estudos também permitem a constatação de fatores que pioram a adesão ao tratamento, divididos em fatores socioeconômicos: residir nas regiões norte, nordeste e centro-oeste,$^{13}$ pobreza, ${ }^{30}$ e pertencer às classes econômicas C/D/E; ${ }^{31}$ demográficos: sexo masculino $0^{13}$ e mulheres; ${ }^{32}$ fatores relacionados ao paciente: hábito tabágico, ${ }^{13}$ esquecimento, ${ }^{18,19}$ solidão, ignorância da medicação, ${ }^{30}$ diminuição da capacidade cognitiva ${ }^{33}$ e mental, ${ }^{23}$ comprometimento funcional ${ }^{183}$ e menor número de comorbidades, ${ }^{23}$ depressão ${ }^{32-33-34}$ e transtorno mental, ${ }^{31}$ resistência ao tratamento e inatividade física, ${ }^{32}$ conhecimento insatisfatório sobre a doença ${ }^{35,36}$ e má adesão à dieta e atividade física; ${ }^{37}$ relacionados à terapêutica: farmacoterapia complexa, ${ }^{35}$ início do tratamento há menos de três meses, ${ }^{31,38}$ custos medicamentos ${ }^{33}$ e experiências prévias com 


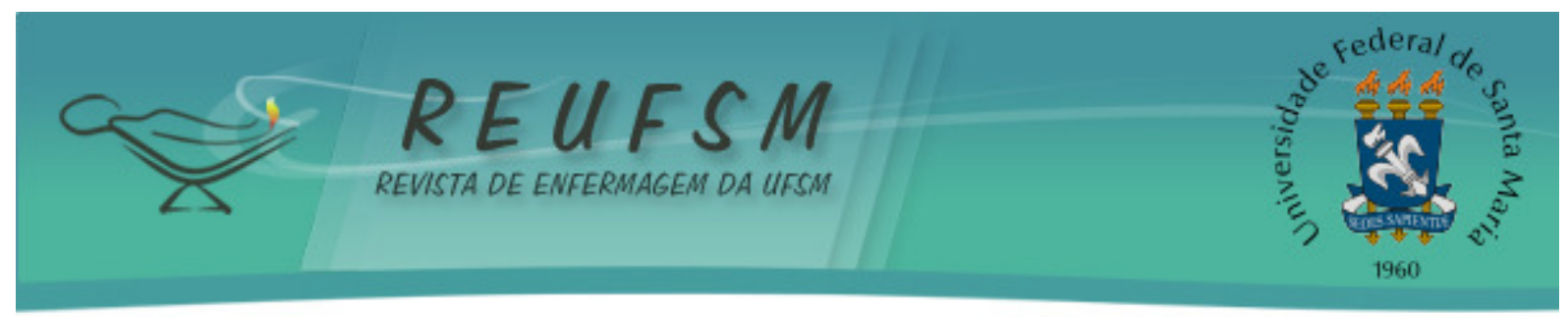

efeitos colaterais; ${ }^{18-19,33}$ relacionados ao sistema de saúde: insatisfação com o serviço prestado $^{35}$ e medicações indisponíveis pelo sistema de saúde. ${ }^{36}$

Os estudos demonstram que não há concordância sobre o fato de a adesão ser melhor entre os homens e em indivíduos mais jovens, ${ }^{13,15,27}$ nem em relação à etnia ou comorbidades. ${ }^{17,38}$ Tais divergências apontam a necessidade de atenção por parte dos enfermeiros em individualizar suas intervenções, independente do sexo e idade, fato que vai de encontro ao estudo ${ }^{39}$ em que variáveis sociodemográficas e clínicas podem não predizer a adesão ao tratamento. Corroborando com o fator idade, no estudo em que a aposentadoria esteve associada com pior adesão, cabe ressaltar que $48 \%$ tinham acima de 60 anos, o que pode minimizar o efeito isolado da aposentaria, neste caso a pior adesão pode ter sido em decorrência da idade mais avançada. ${ }^{30} \mathrm{~A}$ complexidade da farmacoterapia ${ }^{30}$ se constitui em um importante fator para a não adesão ao tratamento medicamentoso pelas questões gerais de acesso, como distribuição precária e precificação, ${ }^{30}$ ou por oscilações no uso quando da responsabilidade do próprio doente, seja por esquecimento ${ }^{30}$ ou por horários irregulares. ${ }^{35}$

No presente estudo ainda verificamos que a hipertensão e diabetes estão associadas. Do total de estudos incluídos, em $23 \%$ os pacientes possuem ambas as doenças $^{14,17,19,23,28,34}$ o que incrementa a complexidade das medidas e, principalmente, do manejo farmacológico, tendo em vista que aumenta a quantidade de fármacos prescritos assim como a necessidade de intensificação das medidas não-farmacológicas.

0 papel da enfermagem ganha relevância por ser uma das pontes entre a comunicação da instituição de saúde com o paciente e a família. ${ }^{40} \mathrm{Um}$ estudo realizado em $2008,{ }^{41}$ enfatiza que os subsídios para a enfermagem não atingem o ideal aprofundamento que pode conferir mudança de comportamento a cada paciente. Nesse sentido fica evidente o desafio para as intervenções de enfermagem diante da complexidade de fatores que estão imbuídos na adesão ao tratamento farmacológico e não farmacológico. 0 enfermeiro necessita se apropriar de diversos fatores que exercem influencia nas atitudes do paciente com HAS e DM, dentre elas, alterações de estilo de vida, tomada correta dos medicamentos e orientações sobre a doença e o tratamento. Colaborando, assim, para melhorar os índices de adesão entre os pacientes com doença crônica.

\section{CONCLUSÃO}

0 presente estudo identificou que os fatores que melhoram e pioram a adesão ao tratamento de pacientes com hipertensão e diabetes são multifatoriais e relacionados às variáveis do próprio paciente, do tratamento em si e do sistema de saúde. Tais fatores direcionam a reflexão sobre as possíveis intervenções de enfermagem que podem melhorar os índices de adesão às medidas terapêuticas e, por fim, atenuar as complicações destas doenças. Pode-se, portanto, visualizar mudanças, por meio de intervenções de enfermagem que valorizem o ambiente familiar, atividades de grupo, orientações quanto às mudanças de estilo de vida, conhecimento da doença e sobre esquema medicamentoso menos complexo.

Porém, os estudos não abordaram os fatores que pioram a adesão ao tratamento sob a perspectiva dos profissionais de enfermagem, gerando, portanto, uma lacuna na literatura quanto às possíveis intervenções que possam ser eficazes na melhor adesão ao tratamento farmacológico ou não. Novos estudos são necessários no sentido de avaliar a eficácia das propostas do Ministério da Saúde no que tange a HAS e DM2 e, consequentemente, efetivando o cuidado pautado no diálogo e adaptado a cada indivíduo no seu contexto de vida. 


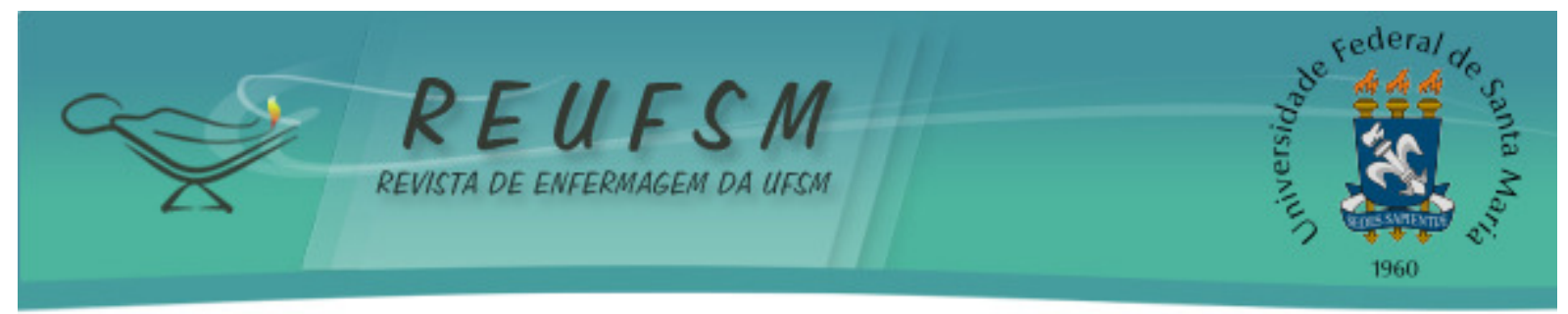

\section{REFERÊNCIAS}

1. Brasil. Ministério da Saúde. Secretaria de Vigilância em Saúde. Departamento de Análise de Situação de Saúde. Plano de Ações Estratégicas para o enfrentamento das Doenças Crônicas Não Transmissíveis (DCNT) no Brasil 2011-2022. Brasília (DF): Ministério da Saúde; 2011.

2. Sociedade Brasileira de Cardiologia, Sociedade Brasileira de Hipertensão, Sociedade Brasileira de Nefrologia. VI Diretrizes Brasileiras de Hipertensão. Arq Bras Cardiol. 2010;95(1 Supl 1):1-51.

3. World Health Organization (WHO). Adherence to long-term therapies: evidence for action. Genebra; 2003 [acesso em 2016 mar 25]. Disponível em: http://www.who.int/chp/knowledge/publications/adherence_report/en/.

4. Girotto E, Andrade SM, Cabrera MAS, Matsuo T. Adesão ao tratamento farmacológico e não farmacológico e fatores associados na atenção primária na hipertensão arterial. Ciênc Saúde Colet [Internet]. 2013 [acesso em 2016 jun 01];18(6):1765-72. Disponível em: http://www.scielosp.org/pdf/csc/v18n6/27.pdf.

5. Oingman W. Métodos de avaliação da adesão ao tratamento anti-hipertensivo. Rev Bras Hipertens [Internet]. 2006 jan [acesso em 2016 dez 25];13(1):[04 páginas]. Disponível em: http://departamentos.cardiol.br/dha/revista/13-1/08-metodos-de-avaliacao.pdf.

6. Gomes-Villas Boas LC, Lima MLSA, Pace AE. Adesão ao tratamento do diabetes mellitus: validação de instrumentos para antidiabéticos orais e insulina. Rev Latinoam Enferm [Internet]. 2014 jan-fev [acesso em 2016 jun 01];22(1):[08 páginas]. Disponível em: DOI: 10.1590/0104-1169.3155.2386. http://www.scielo.br/pdf/rlae/v22n1/pt_0104-1169-rlae22-01-00011.pdf.

7. Boulé NG, Haddad E, Kenny GP, Wells GA, Sigal RJ. Effects of exercise on glycemic control and body mass in type 2 Diabetes Mellitus: a meta-analysis of controlled clinical trials. JAMA. 2001 set;286(10):1218-27.

8. Boulé NG, Kenny GP, Haddad E, Wells GA, Sigal RJ. Meta-analysis of the effect of structured exercise training on cardiorespiratory fitness in type 2 Diabetes Mellitus. Diabetologia. 2003 aug;46(8):1071-81.

9. Mendes KDS, Silveira RCCP, Galvão CR. Revisão integrativa: método de pesquisa para a incorporação de evidências na saúde e na enfermagem. Texto \& Contexto Enferm [Internet]. 2008 out [acesso em 2015 dez 18];17(4):758-64. Disponível em: http://www.scielo.br/pdf/tce/v17n4/18.pdf.

10. Brasil. Ministério da Saúde. Secretaria de Atenção à Saúde. Departamento de Atenção Básica. Hipertensão arterial sistêmica. Brasília (DF): Ministério da Saúde; 2006. (Caderno de Atenção Básica; 15). (Série A. Normas e Manuais Técnicos).

11. Brasil. Ministério da Saúde. Departamento de Atenção Básica. Estratégias para o cuidado da pessoa com doença crônica: diabetes mellitus. Brasília (DF): Ministério da Saúde; 2013. (Cadernos de Atenção Básica; 36).

12. Galvão CM, Sawada NO, Mendes IAC. A busca das melhores evidências. Rev Esc Enferm USP [Internet]. 2003 [acesso em 2016 jun 01];37(4):[07 páginas]. Disponível em http://www.scielo.br/pdf/reeusp/v37n4/05.pdf.

13. Ferreira RA, Barreto SM, Giatti L. Hipertensão arterial referida e utilização de medicamentos de uso contínuo no Brasil: um estudo de base populacional. Cad Saúde 


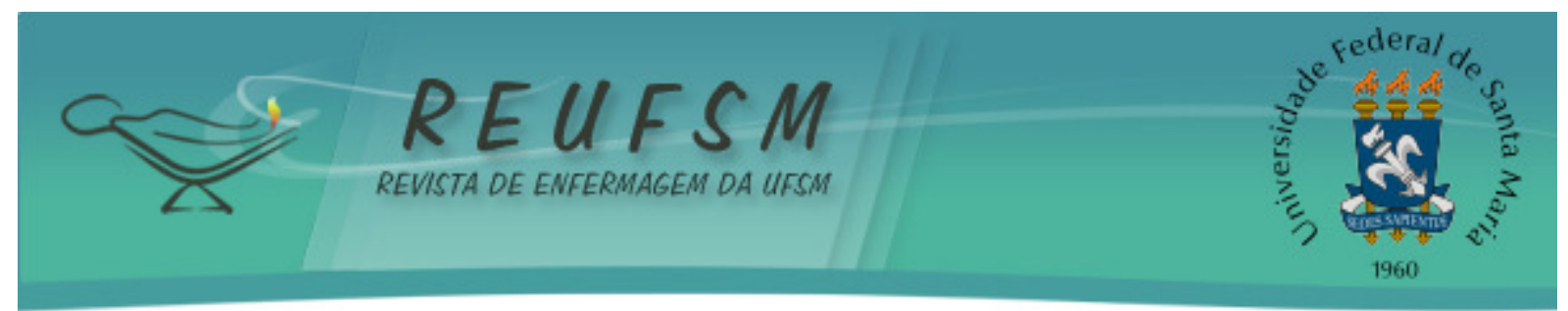

Pública [Internet]. 2014 abr [acesso em $2015 \mathrm{dez}$ 18];30(4):815-26. Disponível em: http: //www.scielo.br/pdf/csp/v30n4/0102-311X-csp-30-4-0815.pdf.

14. Schroeder EB, Hanratty R, Beaty BL, Bayliss EA, Havranek EP, Steiner JF. Simultaneous control of diabetes, hypertension, and hyperlipidemia in two health systems. Circ Cardiovasc Qual Outcomes [Internet]. 2012 set [acesso em 2015 dez 18];5(5):[8 páginas]. Disponível em: http://circoutcomes. ahajournals.org/content/5/5/645. full.pdf+html.

15. Ribeiro ÍJS; Boery RNS O, Casotti CA, Freire IV, Teixeira JRB, Boery EN. Prevalência e fatores associados à adesão ao tratamento medicamentoso por pacientes com hipertensão arterial. Rev Baiana Enferm [Internet]. 2015 jul-set [acesso em 2016 ago 20];29(3):250-60. Disponível em: http://search.proquest.com/openview/5f73f51f9c70bc418c635c88568f0d32/1?pqorigsite=gscholar\&cbl=2040112.

16. Girotto E, Andrade SM, Cabrera MAS, Matsuo T. Adesão ao tratamento farmacológico e não farmacológico e fatores associados na atenção primária da hipertensão arterial. Ciênc Saúde Colet [Internet]. 2013 jun [acesso em 2015 dez 18];18(6):1763-72. Disponível em: http: //www.scielo.br/pdf/csc/v18n6/27.pdf.

17 Nataraja N, Putnam W, Aarsen KV, Lawson B, Burge F. Adherence to antihypertensive medications among family practice patients with diabetes mellitus and hypertension. Can Fam Physician [Internet]. 2013 fev [acesso em 2015 dez 18];59(2):[7 páginas]. Disponível em: http://www.cfp.ca/content/59/2/e93.full.pdf+html.

18. Al-Ramahi R. Adherence to medications and associated factors: a cross-sectional study among Palestinian hypertensive patients. J Epidemiol Glob Health [Internet]. 2015 jun [acesso em 2016 ago 20];5(2):[7 páginas]. Disponível em: http: / /linkinghub.elsevier.com/retrieve/pii/S2210-6006(14)00053-7.

19. Varleta P, Akel C, Acevedo M, Salinas C, Pino J, Opazo V, et al. Assessment of adherence to antihipertensive therapy. Rev Med Chil [Internet]. 2015 mai [acesso em 2016 ago 20];143(5):569-76. Disponível em: http://www.scielo.cl/pdf/rmc/v143n5/art03.pdf.

20. Vacek JL, Hunt SL, Shireman T. Hypertension medication use and adherence among adults with developmental disability. Disabil Health J [Internet]. 2013 out [acesso em 2015 dez 18];6(4):297-302. Disponível em: http://ac.els-cdn.com/S1936657413000435/1-s2.0S1936657413000435-main.pdf?_tid=49c49b6a.

21. Vitor AF, Monteiro FPM, Morais HCC, Vasconcelos JDP, Lopes MVO, Araujo TL. Perfil das condições de seguimento terapêutico em portadores de hipertensão arterial. Esc Anna Nery Rev Enferm [Internet]. 2011 abr-jun [acesso em $2015 \mathrm{dez}$ 19];15(2):251-60. Disponível em: http://www.scielo.br/pdf/ean/v15n2/v15n2a06.pdf.

22. Crowley MJ, Grubber JM, Olsen MK, Bosworth HB. Factors associated with nonadherence to three hypertension self-management behaviors: preliminary data for a new instrument. J Gen Intern Med [Internet]. 2012 ago [acesso em 2015 dez 19];28(1):99-106. Disponível em: http: //link.springer.com/article/10.1007\%2Fs11606-012-2195-1.

23. Gutierrez-Angulo ML, Lopetegi-Urangaa P, Sanchez-Martín I, Garaigordobil-Landazabal M. Cumplimiento terapéutico en pacientes con hipertensión arterial y diabetes mellitus 2 . Rev Calid Asist [Internet]. 2012 mar-abr [acesso em 2015 dez 19];27(2):[5 páginas]. Disponível em: http://www.elsevier.es/es-revista-revista-calidad-asistencial-256-pdfS1134282X11001722-S300.

24. Ribeiro AG, Ribeiro SMR, Dias CMGC, Ribeiro AQ, Castro FAF, Varela MMS, et al. Nonpharmacological treatment of hypertension in primary health care: A comparative clinical 


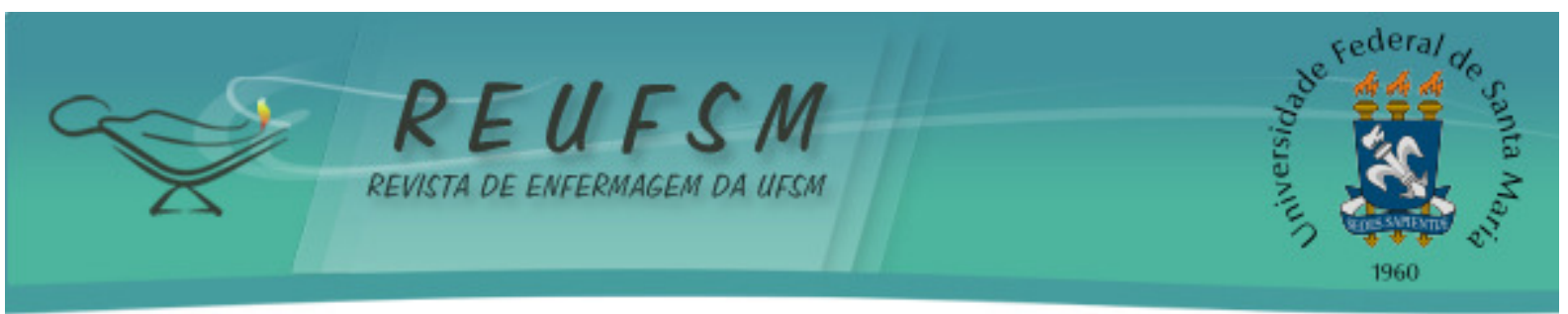

trial of two education strategies in health and nutrition. BMC Public Health [Internet]. 2011 ago [acesso em 2015 dez 19];11:[10 páginas]. Disponível em: http://www.ncbi.nlm.nih.gov/pmc/articles/PMC3171370/pdf/1471-2458-11-637.pdf.

25. Alhalaiqa F, Kho D, AH N, Clark A, Gray R. Adherence therapy for medication noncompliant patients with hypertension: a randomised controlled trial. J Hum Hypertens [Internet]. 2012 fev [acesso em 2015 dez 19];26(2):117-26. Disponível em: http://www.ncbi.nlm.nih.gov/pmc/articles/PMC3257548/pdf/jhh2010133a.pdf.

26. Hedna K, Hakkarainen KM, Gyllensten H, Jönsson AK, Andersson Sundell K, Petzold M,et al. Adherence to antihypertensive therapy and elevated blood pressure: should we consider the use of multiple medications? PLos One [Internet]. 2015 set [acesso em 2016 ago 20];10(9):[14 páginas]. Disponível em https://www.ncbi.nlm.nih.gov/pmc/articles/PMC4567373/pdf/pone.0137451.pdf.

27. Faria HTG, Santos MA, Arrelias CCA, Rodrigues FFL, Gonela JT, Teixeira CRS, et al. Adesão ao tratamento em diabetes mellitus em unidades da Estratégia Saúde da Família. Rev Esc Enferm USP [Internet]. 2014 abr [acesso em 2015 dez 19];48(2):257-63. Disponível em: http://www.scielo.br/pdf/reeusp/v48n2/pt_0080-6234-reeusp-48-02-257.pdf.

28. Fürthauer J, Flamm M, Sönnichsen A. Patient and physician related factors of adherence to evidence based guidelines in diabetes mellitus type 2, cardiovascular disease and prevention: a cross sectional study. BMC Fam Pract [Internet]. $2013 \mathrm{fev}$ [acesso em 2015 dez 19];14:[8 páginas]. Disponível http: / / bmcfampract.biomedcentral.com/track/pdf/10.1186/1471-2296-1447? site=bmcfampract. biomedcentral.com.

29. Solomon A, Schoenthaler A, Seixas A, Ogedegbe G, Jean-Louis G, Lai D. Medication routines and adherence among hypertensive African Americans. J Clin Hypertens (Greenwich) [Internet]. 2015 set [acesso em 2016 ago 20];17(9):668-72. Disponível em: https: //www.ncbi.nlm.nih.gov/pmc/articles/PMC4654115/pdf/nihms-736749.pdf.

30. Karakurt $P$, Kasikçi M. Factors affecting medication adherence in patients with hypertension. J Vasc Nurs [Internet]. 2012 dez [acesso em 2015 dez 19];30(4):18-26. Disponível em: http://www.jvascnurs.net/article/S1062-0303(12)00/pdf.

31. Santa-Helena ET, Nemes MIB, Neto JE. Fatores associados à não-adesão ao tratamento com anti-hipertensivos em pessoas atendidas em unidades de saúde da família. Cad Saúde Pública [Internet]. $2010 \mathrm{dez}$ [acesso em $2015 \mathrm{dez}$ 19];26(12):2389-98. Disponível em: http: //www.scielo.br/pdf/csp/v26n12/17.pdf.

32. Irvin MR, Shimbo D, Mann DM, Reynolds K, Krousel-Wood M, Limdi NA, et al. Prevalence and correlates of low medication adherence in apparent treatment resistant hypertension. J Clin Hypertens (Greenwich) [Internet]. 2012 out [acesso em 2015 dez 19];14(10):[6 páginas]. Disponível em: http://www.ncbi.nlm.nih.gov/pmc/articles/PMC3464920/pdf/nihms388509.pdf.

33. Bogner HR, Morales KH, Vries HF, Cappola AR. Integrated management of type 2 diabetes mellitus and depression treatment to improve medication adherence: a randomized controlled trial. Ann Fam Med [Internet]. 2012 jan-fev [acesso em 2015 dez 19];10(1):[8 páginas]. Disponível em: http://www.ncbi.nlm.nih.gov/pmc/articles/PMC3262455/pdf/0100015.pdf.

34. Klamerus M, Kerr EA, Bosworth HB, Schmittdiel J, Heisler M. Characteristics of diabetic patients associated with achieving and maintaining blood pressure targets in the adherence and intensification of medications program. Chronic Illn [Internet]. 2014 mar [acesso em 


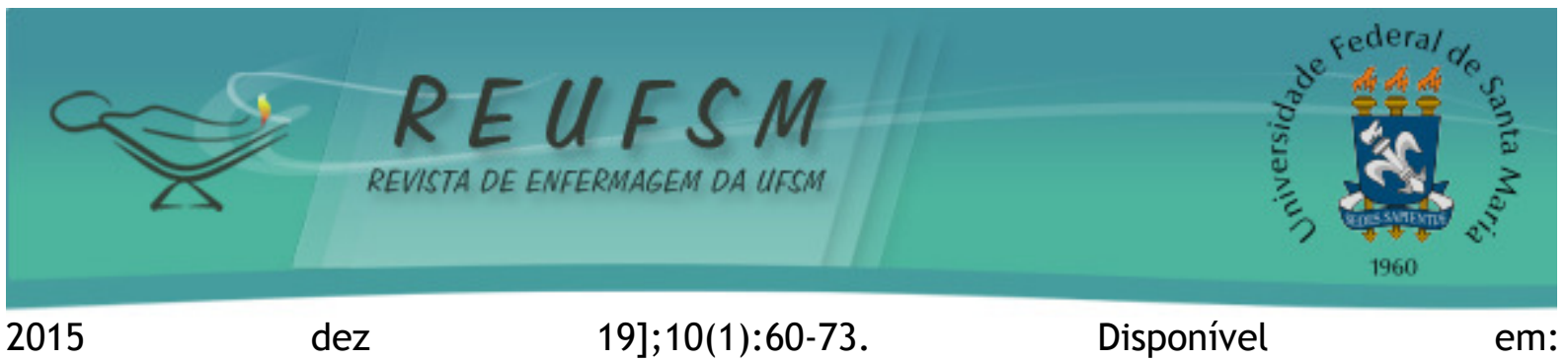

http://www.ncbi.nlm.nih.gov/pmc/articles/PMC4134130/pdf/nihms585426.pdf.

35. Barreto MS, Reiners AAO, Marcon SS. Conhecimento sobre hipertensão arterial e fatores associados a não adesão à farmacoterapia. Rev Latinoam Enferm [Internet]. 2014 maio-jun [acesso em 2015 dez 19];22(3):484-90. Disponível em: http://www.scielo.br/pdf/rlae/v22n3/pt_0104-1169-rlae-22-03-00491.pdf.

36. Lulebo AM, Mutombo PB, Mapatano MA, Mafuta EM, Kayembe PK, Ntumba LT, et al. Predictors of non-adherence to antihypertensive medication in Kinshasa, Democratic Republic of Congo: a cross-sectional study. BMC Res Notes [Internet]. 2015 out [acesso em 2016 ago 20]; 8(526):[8 páginas]. Disponível em: https://www.ncbi.nlm.nih.gov/pmc/articles/PMC4591704/pdf/13104_2015_Article_1519.pdf.

37. Walker RJ, Smalls BL, Hernadez-Tejada MA, Campbell JA, Davis KS, Egede LE, et al. Effect of diabetes fatalism on medication adherence and self-care behaviors in adults with diabetes. Gen Hosp Psychiatr [Internet]. 2012 nov-dez [acesso em 2015 dez 19];34(6):598$603 . \quad$ Disponível em: http://www.ncbi.nlm.nih.gov/pmc/articles/PMC3479321/pdf/nihms395592.pdf.

38. Blair IV, Steiner JF, Hanratty R, Price DW, Fairclough DL, Daugherty SL, et al. An investigation of associations between clinicians' ethnic or racial bias and hypertension treatment, medication adherence and blood pressure control. J Gen Intern Med. 2014;29(7):987-95.

39. Gomes-Villas Boas LC, Foss MC, Foss-Freitas MCF, Torres HC, Monteiro LZ, Pace AE. Adesão à dieta e ao exercício físico das pessoas com diabetes mellitus 1 . Texto \& Contexto Enferm [Internet]. 2011 abr-jun [acesso em 2016 jun 01];20(2):[07 páginas]. Disponível em: http://www.scielo.br/pdf/tce/v20n2/a08v20n2.

40. Gomes-Villas Boas LC, Foss MC, Freitas MCF, Pace AE. Relação entre apoio social, adesão aos tratamentos e controle metabólico de pessoas com diabetes mellitus. Rev Latinoam Enferm [Internet]. 2012 jan-fev [acesso em 2015 dez 19];20(1):[8 páginas]. Disponível em: http://www.scielo.br/pdf/rlae/v20n1/pt_08.pdf.

41. Reiners AA, Azevedo RC, Vieira MA, Arruda AL. Produção bibliográfica sobre adesão/não-adesão de pessoas ao tratamento de saúde. Ciênc Saúde Colet [nternet]. 2008 dez [acesso em 2015 dez 19];13(2):2299-306. Disponível em: http://www.scielo.br/pdf/csc/v13s2/v13s2a34.pdf.

Data de recebimento: 06/08/2015

Data de aceite: 05/09/2016

Autor responsável: Michelli Cristina Silva de Assis.

Endereço postal: Rua Roque Gonzales, n. 177 ap. 205. Bairro Jardim Botânico. Porto Alegre - RS. CEP: 90690-270. Brasil.

Email: michellicassis@gmail.com 